\title{
Clinical anatomy of the upper limb: an overview
}

\author{
Bruno Grignon $^{1} \cdot$ Fabrice Duparc ${ }^{2} \mathbb{D}$
}

Published online: 28 April 2021

(c) The Author(s), under exclusive licence to Springer-Verlag France SAS, part of Springer Nature 2021

In 2021, a better knowledge and/or understanding of numerous anatomical topics regarding the upper limb may still be of great interest to improve medical, radiologic, and surgical every-day practice.

Among them, anatomical variants are sometimes crucial both for diagnosis and treatment of intercurrent disorders of adjacent structures. They may also be useful to avoid iatrogenic lesions and understand unusual clinical signs. In addition, they must be well-known and recognized before any surgical procedure in the case of structures such tendons that can be used in both orthopedic and reconstructive surgery.

Some of these important aspects are presented and discussed in this issue.

With an incidence of $2.5 \%$, the axillary arch (a fleshy slip running from the latissimus dorsi to the anterior aspect of the humerus, the origin of which being still debated) must be known by all plastic and oncological surgeons dealing with breast cancer.

The clinical consequences of variations of the pectoralis minor muscle are generally minor, but they also may contribute to thoracic outlet syndrome, subcoracoid or subacromiaL impingement, adhesive capsulitis, dyskinesia, or even SLAP (superior labral anterior to posterior) lesions.

Anatomical variations of the coraco-brachialis muscle, the flexor pollicis longus muscle, and the pronator teres muscle, which may be involved in musculocutaneous and median neuropathy, are also reported in this issue.

Conversely, anatomic variants of these nerves are also presented, sometimes resulting in potential entrapment risks,

Fabrice Duparc

fabrice.duparc@univ-rouen.fr

Bruno Grignon

b.grignon@chu-nancy.fr

1 Department of Anatomy, Faculty of Medicine, University of Lorraine, Avenue de la Forêt de Haye, 54505 Vandœuvre-lès-Nancy, France

2 Department of Anatomy, Faculty of Medicine-Pharmacy, Rouen-Normandy University, 22 Boulevard Gambetta, 76183 Rouen, France but also of paramount interest in case of anesthetic blocks, or post-traumatic evaluations on nerve repair.

Besides anatomical variations, critical sites of neurologic compression, such as the entrance of the supinator tunnel (arcade de Frohse), may also deserve a comprehensive and evidence-based study, which is presented in this issue.

Variations in the extensor compartment of forearm and hand, as well as those of palmaris longus, are reported and discussed in the light of their potential interest in reconstructive surgery.

Osseous variations are also sometimes of great value in the pathogenesis and consequently in potential surgical decision-making in various entrapment neuropathies, as is the case for the medial epicondyle in the cubital tunnel syndrome that is the second most common one of the upper limb.

A special attention should be paid to very rare other osseous variations, sometimes misdiagnosed as a fracture, as reported in a case of bipartite lunate in this number.

By contrast, constant relationship between anatomical structures may also be observed and highlighted because of their practical applications. The example of the radial nerve and the lateral border of the triceps aponeurosis, presented in this issue, allow to define a "safe area" for soft-tissue dissection while surgically approaching distal humeral fractures.

Finally, at the cross-road of anatomy, imaging, and physiology, special mention was given to shear wave elastography, exploring the infraspinatus muscle and offering new insights into its functions, as well as the flexor carpi radialis brevis muscle (another anatomical variant).

Wishing you a pleasant reading,

Publisher's Note Springer Nature remains neutral with regard to jurisdictional claims in published maps and institutional affiliations. 